\title{
Tratamento Farmacológico das Alterações Comportamentais e de Humor Decorrentes de Lesões Cerebrais
}

\author{
Sonia M. Dozzi Brucki* \\ Paula A. Rodrigues de Gouveia** \\ Sílvia A. Prado Bolognani** \\ Orlando F. Amodeo Bueno***
}

\begin{abstract}
RESUMO
As lesões cerebrais podem apresentar-se como déficits neurológicos bem evidentes, tais como dificuldades motoras; porém outros déficits menos diagnosticados e valorizados podem estar associdados, como alterações comportamentais e cognitivas. Essas alterações também necessitam de medicação efetiva, tanto para a melhora funcional quanto para possibilitar um convívio social mais adequado. Neste trabalho, revisamos os principais déficits não cognitivos e as principais linhas de tratamento medicamentoso ora em uso.
\end{abstract}

\section{UNITERMOS}

Lesão cerebral, tratamento farmacológico, distúrbio de comportamento.
Doutora em Medicina. Neurologista do Serviço de Reabilitação Neuropsicológica do Centro Paulista de Neuropsicologia CPN - Departamento de Psicobiologia EPM - Unifesp.

** Psicóloga do Serviço de Reabilitação Neuropsicológica do CPN - Departamento de Psicobiologia - EPM - Unifesp.

*** Professor-adjunto, Coordenador Geral do CPN - Departamento de Psicobiologia EPM - Unifesp.

\section{NTRODUÇÃO}

Alterações não cognitivas ocorrem com muita frequiência entre portadores de lesões cerebrais, seja de origem vascular, tumoral ou traumática. Tentaremos expor de forma simplificada e esquemática. Existe uma grande diversidade de sintomas, dependentes do tipo e da localização das lesões, com vários sistemas de neurotransmissores envolvidos. As alterações não cognitivas podem ser exemplificadas por depressão, apatia, ansiedade, agressividade, agitação e delírios. Geralmente, ao nos depararmos com essas alterações, devemos, inicialmente, determinar qual o problema a ser abordado primordialmente, pois pode surgir mais de uma alteração em um mesmo paciente. Outro ponto de fundamental importância no tratamento farmacológico são os efeitos indesejáveis, principalmente dos sedativos e dos que afetam as funções cognitivas. Também é preciso ter cautela com a titulação das doses, já que em indivíduos com lesão cerebral, esses efeitos podem ser observados mais precoce e intensamente. Além dessas considerações iniciais, devemos observar mais atentamente o paciente idoso, pelas alterações na farmacocinética das drogas que ocorrem com o envelhecimento. O processo de metabolização das drogas pode diminuir com a idade e sua distribuição pode ser alterada pelo aumento da razão gordura/água, gerando um aumento na distribuição de substâncias lipofílicas. Os metabólitos também podem estar aumentados por decréscimo da filtração glomerular ${ }^{1}$.

A seguir, esquematizaremos alternativas terapêuticas farmacológicas para cada tipo de alteração não cognitiva mencionada.

\section{DEPRESSÃO}

A prevalência dos quadros depressivos varia entre os estudos realizados, dependendo dos critérios adotados, do tipo de questionário (auto-referido 
ou por familiares), da fase da recuperação do quadro de base (se aguda ou crônica) e do tipo de amostra utilizada. Nos traumas cranioencefálicos (TCE) leves, esta varia de $6 \%$ a $39 \%$ dos pacientes, enquanto entre os TCE graves, de $10 \%$ a $77 \%{ }^{2}$. A depressão varia de $5 \%$ a $60 \%$ dos casos, em pacientes com acidentes vasculares cerebrais, sendo mais freqüente naqueles com lesões frontais e à esquerda, aumentando sua gravidade de acordo com a proximidade ao pólo frontal; nos indivíduos com lesões à direita, a depressão parece associar-se à história familiar e às lesões parietais.

Os pesquisadores têm proposto vários mecanismos responsáveis pela irrupção do quadro depressivo ${ }^{3}$ :

- alteração no metabolismo de catecolaminas e de acetilcolina cerebrais;

- distúrbios neuroendócrinos associados ao envolvimento pituitário;

- cortisol sérico elevado;

- alteração da vigília associada à substância reticular ativadora ascendente.

A escolha da medicação antidepressiva deve ser feita visando a uma interação com outras drogas eventualmente usadas, doenças concomitantes (como hipotiroidismo) e perfil de efeitos colaterais. É importante afastar medicações que induzam à depressão, como inibidores da enzima conversora de angiotensina, betabloqueadores, bloqueadores de canal de cálcio, hipnóticos sedativos, bloqueadores $\mathrm{H} 2$, digoxina e corticóides ${ }^{4}$.

\section{ANTIDEPRESSIVOS TRICÍCLICOS}

Agem na região pré-sináptica, bloqueando a recaptação de noradrenalina (NE) e de serotonina (5-HT), em menor proporção de dopamina (DA). Todos têm efeitos colaterais similares. São agentes antiarrítmicos da classe $1 \mathrm{~A}$, podendo agravar bloqueios cardíacos existentes, e possuem efeito inotrópico negativo. Agentes como a imipramina e a amitriptilina têm maiores efeitos anticolinérgicos, agravando quadros de confusão mental ${ }^{5}$; assim, como possuem ação sedativa, podem ser utilizados quando este é um efeito desejável em um paciente que se apresente agitado. Os pacientes podem exibir também hipotensão postural e ganho de peso. Em indivíduos mais idosos, a escolha mais adequada nessa classe de drogas pode ser a nortriptilina, que possui menor efeito anticolinérgico.

a) Efeitos colaterais

- Anticolinérgicos: boca seca, visão turva, obstipação intestinal e retenção urinária.

- Cardiovasculares: hipotensão postural, aumento da freqüência cardíaca, aumento do intervalo PR e do complexo QRS.
- Neurológicos: confusão mental (mais em idosos), sedação, agitação, tremores de mãos, movimentos coreoatesóticos e acatisia.

- Endócrinos: aumento da secreção de prolactina.

- Cutâneos: exantemas, urticárias, eritema multiforme e fotossensibilidade.

b) Contra-indicações

- Glaucoma de ângulo fechado.

- Bloqueios de ramo, bloqueios de condução e infarto agudo do miocárdio.

c) Inibidores da monoaminooxidase (IMAO)

- Os subtipos da MAO, A e B, estão envolvidos no metabolismo da serotonina, da noradrenalina e da dopamina.

- A moclobemida é um inibidor seletivo da MAO-A e é reversível.

- Pode existir hipotensão postural grave e cuidados precisam ser tomados quanto à dieta, a qual deve ser pobre em tiramina, que é um precursor das catecolaminas, evitando-se crises hipertensivas graves.

d) Inibidores seletivos da recaptação de serotonina (ISRS)

- A deficiência de serotonina tem sido relacionada com depressão, pânico, ansiedade, agressividade e impulsividade, tornando esses inibidores úteis em indivíduos portadores de lesões cerebrais e de distúrbios do comportamento.

- Têm um perfil com menos efeitos colaterais e maior tolerabilidade em comparação aos tricíclicos.

- A potência de inibição é diferente, sendo maior a da sertralina e da paroxetina.

- A farmacocinética é linear (concentração plasmática é proporcional à dose) com a sertralina $\mathrm{e}$ o citalopram, diferentemente da fluoxetina, da paroxetina e da fluvoxamina.

- A fluoxetina parece ter efeito mais ativador, devendo ser dada, preferencialmente, pela manhã, enquanto a paroxetina é mais útil em pacientes ansiosos.

- Podem aumentar ou diminuir a agregação plaquetária, sendo problemáticos em pacientes com acidentes vasculares cerebrais.

- São contra-indicados em indivíduos utilizando terfenadina, astemizol e cisaprida, pelo risco de arritmias cardíacas fatais.

- O uso concomitante de IMAO e de drogas serotoninérgicas ou drogas de ação serotoninérgica pode levar ao aparecimento da síndrome serotoninérgica, caracterizada por ${ }^{1,6}$ : alteração do estado mental e do comportamento (agitação, 
confusão, desorientação, coma); alterações motoras (mioclonias, rigidez, hiper-reflexia, incoordenação); instabilidade autonômica (febre, náuseas, diarréia, diaforese, taquicardia, taquipnéia); mais raramente: febre elevada, crises convulsivas, nistagmo, crises oculógiras, opistótono, disartria, coagulação intravascular disseminada, mioglobinúria, insuficiência renal, arritmias cardíacas, coma e morte.

Atenção ao período de eliminação da droga utilizada antes da substituição medicamentosa por agentes serotoninérgicos.

a) Efeitos colaterais dos ISRS: gastrintestinais: náuseas, vômitos, dores abdominais, diarréia; neurológicos: desordens do movimento (sintomas extrapiramidais e discinesia tardia); psiquiátricos: agitação, insônia, nervosismo, ansiedade; cutâneos: urticária; alterações ponderais: perda de peso com fluoxetina e sertralina (início do tratamento) e aumento de peso com a paroxetina e com o citalopram; borramento visual; anorgasmia

b) Inibidor seletivo da recaptação de serotonina e noradrenalina: venlafaxina

- Tem como metabólito ativo: O-desmetilvenlafaxina.

- Sua discreta ação dopaminérgica pode explicar a melhora, em alguns casos, de alterações cognitivas.

- Efeitos colaterais: náuseas, tonturas, sonolência.

- Pode haver o aparecimento de hipertensão arterial em decorrência da inibição da recaptação da noradrenalina, sendo mais freqüente com doses elevadas, devendo ser realizada uma avaliação do benefício de seu uso em pacientes cardiopatas e hipertensos.

- Pode também precipitar a síndrome neuroléptica maligna.

c) Inibidores de recaptação da serotonina e antagonistas alfa

- Nefazodona: cefaléia, boca seca, náuseas. Cautela em indivíduos cardiopatas, pelo risco de hipotensão postural (cuidado em idosos) e bradicardia. Em idosos, deve ser feita uma titulação com doses menores, devido aos efeitos anticolinérgicos, como confusão mental. Tem propriedades ansiolíticas, podendo ser útil em indivíduos com ansiedade e com depressão.

- Trazodona: hipotensão postural, náuseas, boca seca e sedação (esse efeito pode ser benéfico em pacientes com agitação e insônia). Cuidado com o aparecimento de priapismo (1:1.000 a 1:10.000).

d) Inibidor seletivo da recaptação de dopamina (bupropion)

- É bem tolerado, tem sido utilizado também na dependência à nicotina.
- Embora com efeitos colaterais menos intensos, os mais freqüentes são: agitação, ansiedade, boca seca e obstipação intestinal. Cuidado em pacientes epilépticos, pois aumenta o risco de convulsões.

\section{APATIA}

Os quadros de apatia freqüentemente estão ligados a um alentecimento psicomotor e a um empobrecimento emocional. Interferem de modo crucial nos processos de reabilitação, tornando-se, junto à anosognosia, um dos problemas mais difíceis de se lidar nesses programas. Podem confundir-se com sintomas depressivos. Para a avaliação desses quadros, existe a Apathy Evaluation Scale, que permite diferenciar depressão e ansiedade da apatia ${ }^{7}$. A apatia está muito ligada a lesões frontais e interrupções das vias frontoestriatais e frontomesolímbicas. Recentemente, Kant et $a l .{ }^{8}$ avaliaram casos de TCE e observaram que $10,84 \%$ dos pacientes apresentavam apatia isolada, 10,84\% apresentavam depressão isolada e $60 \%$ exibiam ambos os quadros, concomitantemente. Em indivíduos com $\mathrm{AVC}$, a apatia aparece em aproximadamente $12 \%$ dos casos $^{9}$.

Podem ser utilizadas drogas estimulantes, como metilfenidato e a dextroanfetamina, porém, com cuidado, pois podem piorar quadros confusionais, além de hipertensão e taquicardia, estas últimas devido ao aumento da atividade noradrenérgica. Outra alternativa são os agonistas dopaminérgicos, principalmente para os pacientes com lesões em regiões de gânglios da base ou frontais - a mais utilizada é a bromocriptina. Seus efeitos colaterais mais freqüentes são intolerância gástrica e confusão mental, o que pode ser contornado por uma titulação gradual da medicação.

\section{IRRITABILIDADE}

Esse sintoma caracteriza-se por uma flutuabilidade emocional entre frustração e impaciência, em que o paciente torna-se facilmente perturbado. $\mathrm{O}$ tratamento pode ser realizado observando-se outros sintomas associados. $\mathrm{O}$ uso de bloqueadores beta-adrenérgicos pode ser útil, uma vez que também são efetivos no controle de ansiedade e agitação, além de não terem efeitos colaterais indesejáveis, tais como a sedação excessiva dos benzodiazepínicos. Os mais utilizados são o propranolol e o pindolol. Devem ser evitados em indivíduos com diabetes, asma, doença pulmonar 
obstrutiva crônica e hipertiroidismo. Além disso, podem proporcionar quadros confusionais em indivíduos mais idosos. A diminuição da serotonina tem sido imputada na gênese da irritabilidade, bem como nos comportamentos agressivos. $\mathrm{O}$ uso de agentes serotoninérgicos tem sido estudado nesses pacientes. A sertralina provou sua utilidade no controle desse sintoma no trabalho de Kant et al. ${ }^{10}$. Nesse estudo, entre os 13 pacientes com TCE, $77 \%$ apresentavam irritabilidade.

\section{AGITAÇÃO}

O termo agitação refere-se a vários distúrbios de comportamento: delírios, alucinações, agressões verbais ou físicas, vocalizações ruidosas. São sintomas extenuantes para os familiares ou cuidadores que convivem com o paciente, mesmo quando se manifestam de forma fugaz. A abordagem terapêutica pode ser feita com medicações neurolépticas e não neurolépticas.

Entre as drogas não neurolépticas, podemos utilizar as drogas antiepilépticas:

- Carbamazepina. A carbamazepina é uma das mais utilizadas. Sua ação faz-se pela diminuição da reposição de noradrenalina, de dopamina e do ácido gama-aminobutírico. Sua dose ideal é variável, devendo ser ajustada de acordo com a resposta clínica, devido à variabilidade individual e à interação com outras drogas. Deve ser administrada em três tomadas diárias, sendo permitida sua utilização em duas tomadas, quando prescrevemos a formulação de liberação lenta. Seus efeitos colaterais incluem sonolência, tontura, ataxia, distúrbios visuais e erupções cutâneas. Reações hematológicas graves podem ocorrer, como agranulocitose e anemia aplástica, principalmente em politerapia e em idosos. Além disso, existe o risco de hepatotoxicidade e hiponatremia ${ }^{11}$.

- Valproato. O valproato pode também ser utilizado. Essa droga parece agir sobre o GABA, pelo aumento da atividade da descarboxilase do ácido glutâmico e inibição da GABA transaminase, potencializando a ação pós-sináptica mediada por GABA, à semelhança dos benzodiazepínicos. Os efeitos colaterais mais comuns são náuseas e vômitos. As reações idiossincráticas são preocupantes, entre elas a hepatotoxicidade, assim como a neutropenia e a depressão da medula óssea ${ }^{12}$.

Ainda como não neurolépticos são utilizadas:

- Trazodona - tem bons efeitos devido à sua ação sedativa concomitante à antidepressiva.
- Betabloqueadores - propanolol, pindolol. Observar a titulação da dose para atingir a redução da agitação.

- Buspirona - é uma azapirona, com propriedades agonistas 5-HT1A, útil não só na agitação, como na ansiedade. A vantagem é que não compromete a cognição, além de não apresentar propriedades sedativas; a desvantagem é que o início da ação se dá em 5 a 15 dias $^{13}$.

- Benzodiazepínicos - podem ser utilizados em agitação e ansiedade. Podem piorar quadros confusionais, além de deflagrarem reações paradoxais. A suspensão abrupta pode resultar em aumento da agitação. Deve-se preferir os de curta ação, como lorazepam, oxazepam e temazepam, e aqueles com metabolismo hepático por conjugação, que não diminuem com o envelhecimento, permitindo seu uso seguro em idosos.

Quanto à utilização de medicação neuroléptica, temos que avaliar seus efeitos colaterais mais comuns: sinais de parkinsonismo, acatisia, discinesia tardia. Os neurolépticos mais utilizados eram o haloperidol e a tioridazina, ambos com alta afinidade para os receptores dopaminérgicos D2. A geração atual de neurolépticos caracteriza-se por uma baixa afinidade pelos receptores D2 e aumento da mesma para os receptores serotoninérgicos 5-HT2, com menores efeitos parkinsonianos. Temos com neurolépticos atípicos:

- Clozapina foi o primeiro a aparecer, porém com um perigoso risco de supressão da medula óssea, sendo necessária a monitorização dos glóbulos brancos, semanalmente, por seis meses. A experiência maior é com pacientes parkinsonianos que desenvolveram quadros psicóticos, sem piora da doença com o uso da droga.

- A seguir, foi comercializada a risperidona, que tem menor afinidade pelos receptores D2 do que o haloperidol. Em idosos, porém, doses acima de $2 \mathrm{mg}$ causaram sintomas extrapiramidais semelhantes aos decorrentes do uso de haloperidol.

- A olanzapina já foi utilizada em pacientes parkinsonianos sem piora dos seus sintomas ${ }^{14}$, porém é extremamente cara, em nosso meio.

- Por último, foi lançada a quetiapina, devendo-se aumentar a dose inicial de $25 \mathrm{mg} /$ dia lentamente, a fim de minimizar efeitos adversos como hipotensão postural e sedação.

Como exemplo do uso dessas medicações no meio médico, temos o interessante trabalho de Fugate et al. ${ }^{15}$ em que 129 médicos responderam sobre as drogas utilizadas para tratar agitação em seus pacientes que haviam sofrido TCE. As drogas mais citadas por ordem decrescente de freqüência foram: carbamazepina, 
antidepressivos tricíclicos, propranolol, haloperidol e benzodiazepínicos. Observando-se as respostas dessa amostra de médicos especialistas, a carbamazepina continuava a droga mais utilizada.

\section{CONCLUSÃO}

Quando o tratamento farmacológico de quaisquer desses quadros se faz necessário, devemos refletir em que circunstâncias essas alterações aparecem, sua periodicidade, a interação do paciente com as demais pessoas e inquirir cuidadosamente sobre todas as medicações que o paciente tem usado. Após esses passos, pesar os benefícios e os efeitos colaterais com o tratamento proposto. Quando bem adotadas, essas medidas terapêuticas melhoram o convívio familiar e auxiliam no processo de reabilitação, facilitando essa tarefa. Em nosso Serviço de Reabilitação Neuropsicológica, a abordagem medicamentosa junto às técnicas de reabilitação é comum, sendo adotada, seja por discussões com o próprio médico do paciente ou por acompanhamento neurológico.

\section{SUMMARY}

Pharmacologic Treatment of Humor and Behavioral Disorders in Brain Injured Patients

Brain injured patients could show depression, apathy, psychomotor agitation as well motor deficits. These symptoms impairing the daily activities and social relationships requiring precise medication.

\section{KEYWORDS}

Brain injury, pharmacological treatment, noncognitive disorders.

\section{Referências}

1. Mulchahey JJ, Malik MS, Sabai M et al. Serotonin-selective reuptake inhibitors in the treatment of geriatric depression and related disorders. Int Neuropsychopjarmacol, 2: 121-7, 1999.

2. Silver JM, Yudofsky SC, Hales RE. Depression in traumatic brain injury. Neuropsychiatry, Neuropsychology and Behavioral Neurology, 4:12-23, 1991.

3. Ownsworth TL, Oei TPS. Depression after traumatic brain injury: conceptualization and treatment considerations. Brain Injury, 9:735-51, 1998.

4. Patten SB, Love EJ. Drug-induced depression. Psychoteraphy and Psychosomatics, 66:63-73, 1997.

5. Flint AJ. Recent developments in geriatric psychopharmacology. Can J Psychiatry, 39(suppl. 1): S9-S18, 1994

6. Bodner RA, Lynch $\mathrm{T}$, Lewis $\mathrm{L}$ et al. Serotonin syndrome. Neurology, 45:219-23, 1995.

7. Marin RS. Apathy: a neuropsychiatric syndrome. J Neuropsychiatry and Clinical Neurosciences, 3:243-54, 1991.

8. Kant R, Duffy JD, Pivovarnik A. Prevalence of apathy following head injury. Brain Injury, 12:87-92, 1998.

9. Starkstein SE, Fedoroff JP, Price TR et al. Apathy following cerebrovascular lesions. Stroke, 24:1625-30, 1993.

10. Kant R, Smith-Seemiller L, Zeiler D. Treatment of agression and irritability after head injury. Brain Injury, 12:661-6, 1998.

11. Pellock JM. Carbamazepine side effects in children and adults. Epilepsia, 28(S3):64-70, 1987.

12. Fariello R. In: Levy R, Mattson $\mathrm{RH}$, Penry JK et al. Antiepileptic drugs. $4^{\text {th }}$ ed. NY: Raven Press, 1995.

13. Cadieux RJ. Azapirones: an alternative to benzodiazepines for anxiety. American Family Physician, 53:2349-53, 1996.

14. Wolters EC, Jansen EN, Tuynman-Qua HG et al. Olanzapine in the treatment of dopaminomimetic psychosis in patients with Parkinson's disease. Neurology, 47:1085-7, 1996.

15. Fugate LP, Spacek LA, Kresty LA et al. Measurement and treatment of agitation following traumatic brain injury:Il. A survey of the Brain Injury Special Interest Group of the American Academy of Physical Medicine and Rehabilitation. Arch Phys Med Rehabil, 78:924-8, 1997.

Endereço para correspondência:

Sonia M. D. Brucki

Rua Napoleão de Barros, 925 - Vila Clementino

CEP 04024-012 - São Paulo, SP

E-mail: sbrucki@uol.com.br 